\title{
Mechanistic Insights into the Light-Driven Catalysis of an Immobilized Lipase on Plasmonic Nanomaterials
}

\section{Ribeiro de Barros, Heloise}

2021-01-01

Ribeiro de Barros , H, Garcia , I , Kuttner , C , Zeballos , N , Camargo , P H C , Cordoba de Torresi , S I, Lopez-Gallego , F \& Liz-Marzan , L M 2021, ' Mechanistic Insights into the Light-Driven Catalysis of an Immobilized Lipase on Plasmonic Nanomaterials ' , ACS catalysis , vol. 11 , no. 1 , pp. 414-423 . https://doi.org/10.1021/acscatal.0c04919

http://hdl.handle.net/10138/338157

https://doi.org/10.1021/acscatal.0c04919

acceptedVersion

Downloaded from Helda, University of Helsinki institutional repository.

This is an electronic reprint of the original article.

This reprint may differ from the original in pagination and typographic detail.

Please cite the original version. 


\section{Mechanistic Insights on the Light-Driven Catalysis}

\section{2 of an Immobilized Lipase on Plasmonic}

\section{Nanomaterials}

4 Heloise Ribeiro de Barros, ${ }^{* a, b}$ Isabel García, ${ }^{b, c}$ Christian Kuttner, ${ }^{b}$ Nicoll Zeballos, ${ }^{b}$ Pedro H.

5 C. Camargo, ${ }^{a, d}$ Susana Inés Cordoba de Torresi, ${ }^{a}$ Fernando López-Gallego, ${ }^{*}$ be and Luis M. Liz-

6 Marzán $^{b, c, e}$

7 a Department of Fundamental Chemistry, Institute of Chemistry, University of São Paulo, Av.

8 Prof. Lineu Prestes, 748, Vila Universitária, 05508-000 São Paulo, SP, Brazil.

$9 \quad{ }^{\mathrm{b}}$ CIC biomaGUNE, Basque Research and Technology Alliance (BRTA), Paseo de Miramón

10 182, 20014 Donostia - San Sebastián, Spain.

$11{ }^{\mathrm{c}}$ Centro de Investigación Biomédica en Red, Bioingeniería, Biomateriales y Nanomedicina

12 (CIBER-BBN), Paseo de Miramón 182, 20014 Donostia - San Sebastián, Spain.

$13{ }^{\mathrm{d}}$ Department of Chemistry, University of Helsinki, A.I. Virtasen aukio 1, Helsinki, Finland

$14{ }^{\mathrm{e}}$ Ikerbasque, Basque Foundation for Science, 48013 Bilbao, Spain.

15 KEYWORDS biocatalysis, gold nanostructures, LSPR-enhanced mechanisms, nanotechnology, 16 plasmonic heating, triggered bioactivity. 
18 The use of light as an external stimulus to control enzyme activity is an emerging strategy that

19 enables accurate, remote and noninvasive biotransformations. In this context, immobilization of

20 enzymes on plasmonic nanoparticles offers an opportunity to create light-responsive biocatalytic

21 materials. Nevertheless, a fundamental and mechanistic understanding on the effects of localized

22 surface plasmon resonance (LSPR) excitation over enzyme regulation remains elusive. We

23 investigate herein the plasmonic effects on biocatalysis using Au nanospheres (AuNSp) and

24 nanostars (AuNSt) as model plasmonic nanoparticles, lipase from Candida antarctica fraction B

25 (CALB) as a proof of concept enzyme, and $808 \mathrm{~nm}$ as NIR light excitation. Our data show that

26 LSPR excitation enables an enhancement of 58\% in enzyme activity for CALB adsorbed on

27 AuNSt, compared with the dark conditions. This work shows how photothermal heating over the

28 LSPR excitation enhances CALB activity through favoring product release in the last step of the

29 enzyme mechanism. We propose that the results reported herein shed important mechanistic and

30 kinetic insights in the field of plasmonic biocatalysis and may inspire the rational development of

31 plasmonic nanomaterial-enzyme hybrids with tailored activities under external light irradiation.

33 INTRODUCTION

34 Plasmonic nanomaterials, such as gold nanoparticles (Au NPs), display remarkable optical 35 properties in the visible and near-infrared (NIR) spectral regions. ${ }^{1-3}$ Such properties arise as a result 36 of the excitation of localized surface plasmon resonances (LSPRs). It has been established that 37 LSPR excitation in plasmonic NPs can accelerate a myriad of chemical transformations. ${ }^{4-7}$ This 
38 catalytic effect can occur as a result of the generation of LSPR-excited charge carriers (hot

39 electrons and hot holes) and/or photothermal heating following plasmon decay. ${ }^{4,8,9}$ Surprisingly,

40 only a few studies have explored the use of LSPR excitation to tune biocatalytic reactions. ${ }^{10-15}$

41 The conjugation of enzymes to plasmonic NPs is attractive for applications in biomedicine,

42 such as photothermal therapy ${ }^{16,17}$ and bioimaging, ${ }^{18}$ as well as in chemical manufacturing. ${ }^{13}$ In

43 fact, the use of plasmonic effects at the interface between nanoparticles and enzymes is gaining

44 momentum as a tool to remotely control biocatalytic processes using light as an external

45 stimulus. ${ }^{12,13,19}$ This field, plasmonic biocatalysis, paves the way to tuning enzymes' properties in

46 a non-invasive manner, enabling spatio-temporal control over the biocatalytic processes. ${ }^{12,} 13$

47 Despite these fascinating opportunities, the mechanisms at the "nano-bio" interface underlying the

48 influence of plasmonic effects on enzyme functionality are poorly understood. ${ }^{10,12,20}$

What is already known is that the enzyme/nanomaterial interface plays an important role

50 in the transport of substrates and products from the bulk to the enzyme active site, and vice versa,

51 thereby altering enzyme activity. ${ }^{21}$ For example, recent insightful mechanistic studies revealed that

52 the conjugation of hydrolases (i.e. phosphotriesterase) to quantum dots and Au NPs enhances the

53 enzymatic kinetic efficiency, as compared to their free counterparts. ${ }^{22,}{ }^{23}$ Furthermore, kinetic

54 studies under high viscosity conditions demonstrate that the increase in apparent catalytic rate ( $\mathrm{k}_{\text {cat }}$ )

55 relies on higher product release kinetic constants associated to the last step of the hydrolases

56 catalytic mechanism. Nevertheless, how those kinetic parameters may be altered by light at the

57 interface between enzyme and plasmonic nanomaterials is still an open question that remains

58 largely underexplored. 
To bridge this gap, we report herein on a detailed and systematic study of the effects of

60 LSPR excitation over the activity and enhancement mechanisms in plasmonic biocatalysis.

61 Specifically, we selected the lipase from Candida antarctica fraction B (CALB) as a proof of

62 concept enzyme, whose catalytic mechanism is well understood, Au nanospheres (AuNSp) and

63 nanostars (AuNSt) as model plasmonic NPs, and a NIR laser as the light excitation source. Both

64 on- (AuNSs) and off- (AuNPs) resonance conditions relative to the NIR laser source were

65 investigated to demonstrate the LSPR-driven enhancement effects. Although CALB has been

66 previously conjugated to Au NPs, ${ }^{24-26}$ control over its catalytic activity through plasmonic effects

67 remains elusive. Our data suggest that the localized photothermal heating following LSPR

68 excitation plays an important role toward favoring the reaction step involving product desorption

69 from the biocatalytic active sites, ultimately leading to increased reaction rates.

\section{RESULTS AND DISCUSSION}

The first step toward this study comprised CALB adsorption onto Au NPs. It is well

72 established that enzymes can interact with Au NPs surfaces via the interaction of carboxyl and

73 amine groups present in the amino acid residues of the enzyme structure, following a kinetic

74 process that involves anchoring, crawling, and subsequent binding onto the NPs surface. ${ }^{24,27,28}$ It

75 has also been reported that electrostatic binding can take place between remaining carboxyl groups

76 on the Au NPs'surface (e.g. from citrate employed during synthesis) and amino groups (e.g. Lys

77 and Arg) from the enzyme structure. ${ }^{29}$ Furthermore, enzymes containing thiolated amino acid

78 residues may interact with the Au NPs surface by chemisorption. ${ }^{30,31}$ CALB presents ten thiolated

79 residues in its structure, of which four are methionine and six are cysteine residues, forming three

80 disulfide bonds ${ }^{32}$ and providing favorable conditions for anchoring enzymes onto the NPs

81 surface. $^{27}$ 
AuNSt were synthesized by a seed-mediated growth method, ${ }^{33,}{ }^{34}$ using ascorbic acid as

83 reducing agent, silver nitrate to assist the growth of spiky nanostructures, and CALB as stabilizing

84 molecule. AuNSp were synthesized according to the Turkevich method ${ }^{35}$ and subsequently coated

85 by CALB. Both AuNSt and AuNSp were washed by centrifugation and removal of the supernatant

86 to ensure that only CALB molecules adsorbed onto the NPs surface. AuNSt and AuNSp showed

87 great colloidal stability upon CALB adsorption. The corresponding nanobioconjugates are referred

88 to as AuNSt@CALB and AuNSp@CALB, respectively. Figure 1A-D shows representative TEM

89 images AuNSp@CALB (Figure 1A and B) and AuNSt@CALB (Figure 1C and D). Nanoparticle

90 size distribution histograms are presented in Figure S1. The images confirm the formation of Au

91 nanospheres and nanostars with spiky morphology and sharp tips branching out from a central

92 core. Both AuNSt@CALB and AuNSp@CALB displayed a relatively narrow size distribution,

93 with diameters corresponding to $12 \pm 2$ and $100 \pm 20 \mathrm{~nm}$, respectively. For AuNSt, the tip

94 dimensions were approximately $45 \pm 5 \mathrm{~nm}$ in length and $5 \pm 0.6 \mathrm{~nm}$ in width. Although not

95 conclusive, high-resolution TEM images (Figure 1B and D) evidenced the presence of an organic

96 layer on AuNSp and AuNSt surfaces, which may correspond to adsorbed CALB. The

97 morphologies of AuNSp and AuNSt were thus preserved upon CALB adsorption and no

98 aggregation was observed, even after laser irradiation (Figure S2). This indicates that CALB

99 served as a suitable stabilizing agent for both AuNSp and AuNSt. AuNSp@CALB and

100 AuNSt@CALB exhibited intense LSPR bands around 525 nm and 700 nm, respectively (Figure

101 1E). These LSPR band positions were exploited to study the effect of on and off resonance

102 conditions, relative to the NIR laser wavelength employed in CALB biocatalysis studies $(808 \mathrm{~nm}$,

103 indicated by the dashed line in Figure 1E). 

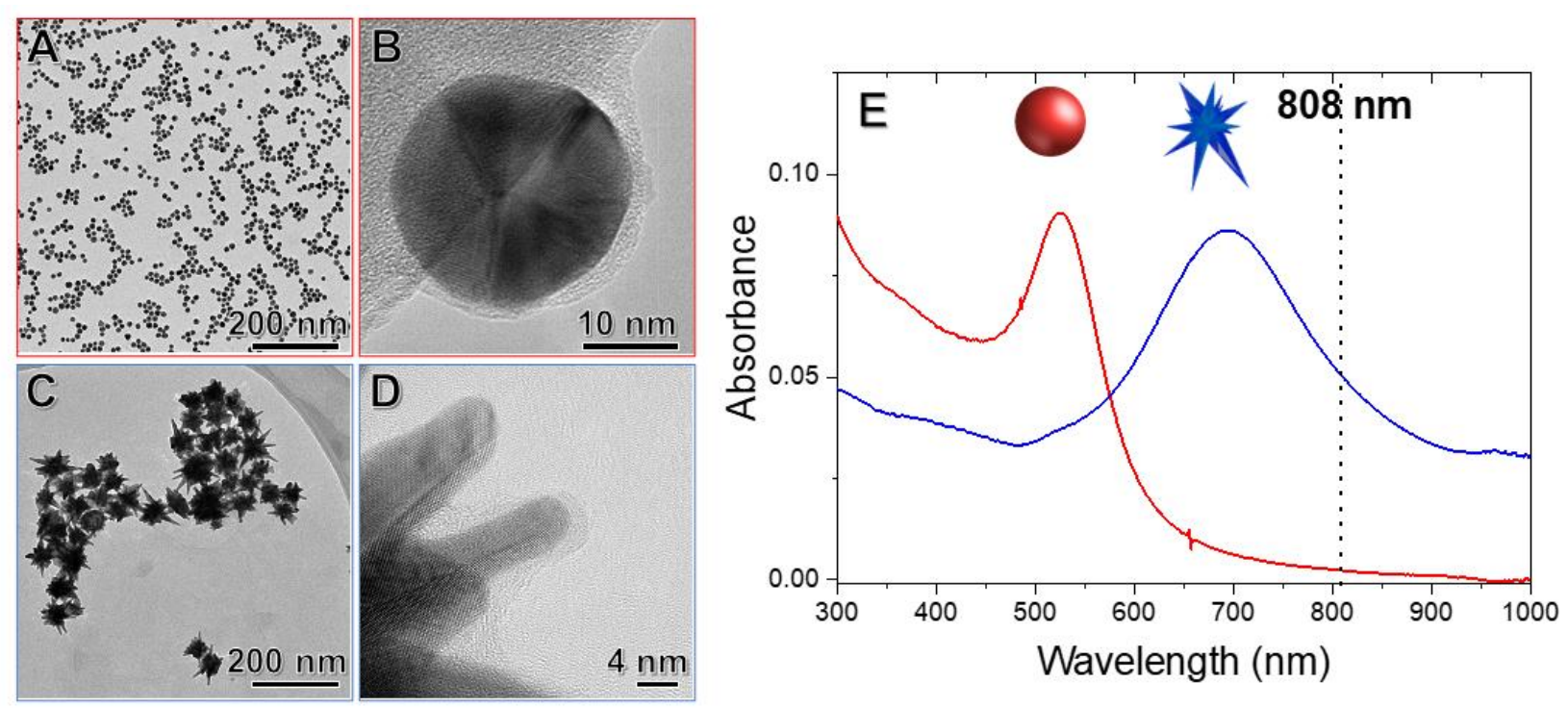

Figure 1. (A-D) TEM (A and C) and high resolution TEM (B and D) images of AuNSp@CALB (A and B) and AuNSt@CALB (C and D). (E) UV-Vis extinction spectra registered from aqueous suspensions containing of AuNSp@CALB (red trace) and AuNSt@CALB (blue trace). The 808 $\mathrm{nm}$ wavelength employed for biocatalysis studies is indicated by the black dashed line.

We then turned our attention to the study of CALB activity toward the hydrolysis of 4nitrophenyl palmitate ( $p$ NPP) as a model reaction (see Scheme 1 in the Experimental section). We

107 found that CALB activity (under light off conditions) decreased upon its adsorption on both AuNSt and AuNSp (Table 1). This behavior is a common trend typically observed for immobilized

109 enzymes, being established that external mass transport restrictions limit their activity. ${ }^{36} \mathrm{We}$ 110 determined the catalytic rate constant $\left(\mathrm{k}_{\mathrm{cat}}\right)$, the binding Michaelis constant $\left(\mathrm{K}_{\mathrm{M}}\right)$, and the catalytic

111 efficiency $\left(\mathrm{k}_{\mathrm{cat}} / \mathrm{K}_{\mathrm{M}}\right)$ of free CALB and the same apparent parameters for the adsorbed enzyme 112 (Table 1 and Figure S3). Under light off conditions, the decrease in $\mathrm{k}_{\text {cat }}$ values for CALB adsorbed 113 on the Au NPs can be related to a partial loss of enzyme activity. Nevertheless, the $\mathrm{K}_{\mathrm{M}}$ values of 114 adsorbed enzymes on the Au NPs were significantly smaller as compared to free CALB. This 115 lower apparent $\mathrm{K}_{\mathrm{M}}$ suggests an increase of substrate local concentration at the NP surface, which 116 causes the higher activities observed at lower bulk substrate concentration. This effect was more 
117 evident for AuNSt than for AuNSp. Similar results were obtained with a homologous lipase from 118 Candida rugosa immobilized on AuNSp. ${ }^{26}$ The $\mathrm{k}_{\mathrm{cat}} / \mathrm{K}_{\mathrm{M}}$ value decreased upon CALB adsorption 119 on the Au NPs, but that decay was 1.7 times lower for AuNSt@CALB than for AuNSp@CALB.

120 The different kinetic behavior of CALB on the two different NP morphologies can be related to 121 the enzyme density for nanoparticles with different curvature, where NPs with a smaller size (i.e., 122 with a higher curvature) display a higher enzyme activity. ${ }^{23}$ In this context, the tips of AuNSt (ca. $1235 \mathrm{~nm}$ in diameter) can provide a surface of much higher curvature, compared to AuNSp (diameter 124 around $20 \mathrm{~nm}$ ), leading to a lower density of CALB at the NP surface that results in a higher 125 enzyme activity.

Table 1. Kinetic parameters determined from Michaelis-Menten plots (Figure S3) for samples 127 under light on and off (dark) conditions ${ }^{a}$.

\begin{tabular}{|c|c|c|c|c|c|c|}
\hline \multirow{2}{*}{ Sample } & \multicolumn{2}{|c|}{$\mathrm{k}_{\mathrm{cat}}\left(\min ^{-1}\right)$} & \multicolumn{2}{|c|}{$\mathrm{K}_{\mathrm{M}}(\mu \mathrm{M})$} & \multicolumn{2}{|c|}{$\mathrm{k}_{\text {cat }} / \mathrm{K}_{\mathrm{M}}\left(\mu \mathrm{M}^{-1} \mathrm{x} \min ^{-1}\right)$} \\
\hline & OFF & ON & OFF & ON & OFF & ON \\
\hline AuNSt@CALB ${ }^{b}$ & $2461 \pm 82$ & $3947 \pm 240$ & $3.2 \pm 0.4$ & $5.1 \pm 0.5$ & $773 \pm 180$ & $765 \pm 448$ \\
\hline $\mathrm{AuNSp} @ \mathrm{CALB}^{c}$ & $2140 \pm 126$ & $2705 \pm 372$ & $4.8 \pm 0.6$ & $7.0 \pm 2.0$ & $443 \pm 192$ & $385 \pm 186$ \\
\hline Free $\mathrm{CALB}^{d}$ & $15855 \pm 732$ & $15966 \pm 758$ & $10.4 \pm 4.3$ & $9.7 \pm 3.9$ & $1520 \pm 169$ & $1640 \pm 192$ \\
\hline
\end{tabular}

\footnotetext{
${ }^{a}$ Reaction conditions: PBS buffer at $\mathrm{pH} 7.4$; at room temperature (approx. $20^{\circ} \mathrm{C}$ ); NIR laser irradiation at $3.2 \mathrm{~W} / \mathrm{cm}^{2}$. Enzyme concentration used: ${ }^{b} 1.5 \mu \mathrm{mol} \mathrm{L}^{-1},{ }^{c} 1.0 \mu \mathrm{mol} \mathrm{L}^{-1}$, and ${ }^{d} 1.1 \mu \mathrm{mol} \mathrm{L}{ }^{-1}$. The kinetic constants calculated for the immobilized enzymes are apparent constants, since they also account for mass transfer restrictions.
}

We further studied the effect of light irradiation on the hydrolytic activity of AuNSt@CALB and AuNSp@CALB under different irradiation conditions. The reactions were carried out in a quartz cuvette illuminated with a NIR laser at $\lambda=808 \mathrm{~nm}$, measuring the release 
135 of 4-nitrophenolate ( $p \mathrm{NP}$ ) in situ, using a UV-Vis spectrophotometer (Figure 2A). Unlike the

136 results under dark conditions, NIR irradiation enhanced the enzymatic activity of AuNSt@CALB

137 to a significantly higher extent than that for AuNSp@CALB irradiated under the different laser

138 powers (Figure $2 \mathrm{~B}$ and Table 1 ). This result agrees with the better match between the incoming

139 light wavelength $(808 \mathrm{~nm})$ and the LSPR position in AuNSt (700 nm, Figure 1E), as compared to

140 AuNSp (525 nm, Figure 1E). In the case of AuNSt@CALB, the activity increases with laser power

141 until reaching a plateau at laser powers above $1.6 \mathrm{~W} / \mathrm{cm}^{2}$. No differences were observed for the

142 activity of free CALB under light on and off conditions (Figure S4). Therefore, NIR irradiation

143 only leads to a significant enhancement on the activity of CALB molecules at the surface of

144 AuNSt, which feature a LSPR position which better matches the light excitation wavelength.

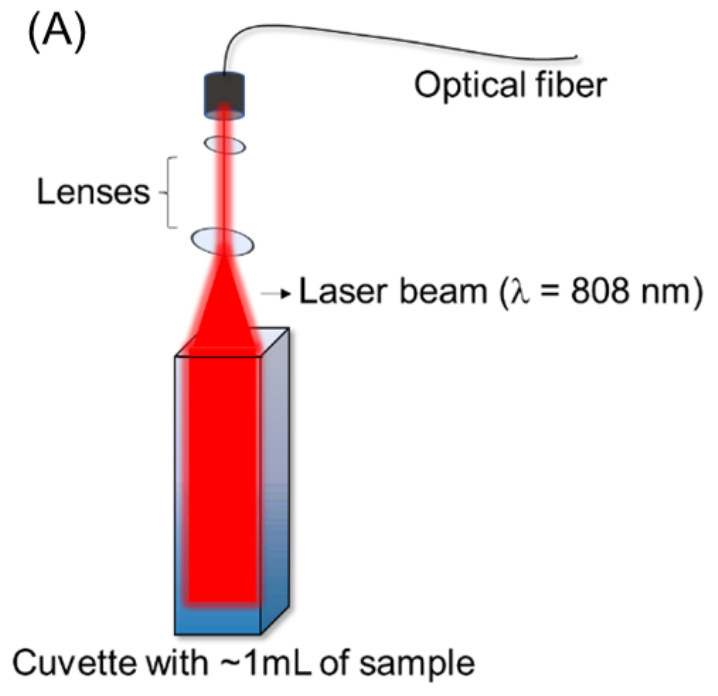

Figure 2. (A) Schematic illustration of the laser irradiation setup. (B) Effect of NIR laser power $(\lambda=808 \mathrm{~nm})$ on the enzymatic activity of AuNSt@CALB (blue squares) and AuNSp@CALB (red circles).

To unravel the effect of LSPR excitation over enzymatic activity, we investigated the

151 heating capacity of AuNSt and AuNSp under the employed light irradiation conditions. The 152 samples were therefore illuminated with the NIR laser and the temperature changes in the colloidal 
153 dispersion over time were monitored with a thermal camera. When the temperature reached 154 thermal equilibrium, the laser was turned off and the cooling down curve was recorded to quantify 155 heat dissipation to the solution. Figure 3A shows exemplary heating and cooling curves for both 156 AuNSt@CALB and AuNSp@CALB. The molar heat transfer rates for both AuNSt and AuNSp 157 were calculated by fitting these temperature time-courses to Equation $1,{ }^{37}$ as illustrated in Figure $1583 \mathrm{~B}$.

$$
\frac{\Delta Q}{c_{A u}}=\frac{Q_{\text {sample }}-Q_{\text {medium }}}{\varepsilon_{400} / 2.4 \text { mmolL }^{-1}}
$$

160 Here, the generated heat output $(\Delta \mathrm{Q})$, obtained from the heat difference between the sample $161\left(\mathrm{Q}_{\text {sample }}\right)$ and the medium $\left(\mathrm{Q}_{\text {medium }}\right)$, is related in terms of the estimated gold concentration $\left(\mathrm{c}_{\mathrm{Au}}=\right.$ $\left.162 \varepsilon_{400} / 2.4 \mathrm{mmol} \mathrm{L}^{-1}\right)^{38}$ in the sample. It was found that the molar heat transfer rate was much larger 163 for AuNSt@CALB than for AuNSp@CALB. In this case, LSPR excitation leads to photothermal 164 heating because of plasmon decay. Such a photothermal heating effect takes place close to the 165 NPs' surface and is further dissipated to the reaction mixture, leading to the detected temperature 166 increase. Our data indicate that AuNSt are more efficient nano-sources of heat ${ }^{8}$ than AuNSp under 167 the employed NIR irradiation conditions. In fact, Figure 3C shows that AuNSt@CALB under NIR 168 irradiation $\left(3.2 \mathrm{~W} / \mathrm{cm}^{2}\right)$ were capable to increase the bulk temperature of the reaction mixture up 169 to $7.3^{\circ} \mathrm{C}$, versus the $2.7^{\circ} \mathrm{C}$ observed for AuNSp@CALB under the same conditions. As expected 170 from the photothermal heating triggered by LSPR excitation, we observed an increase of the bulk 171 temperature by increasing the laser power. 
(A)

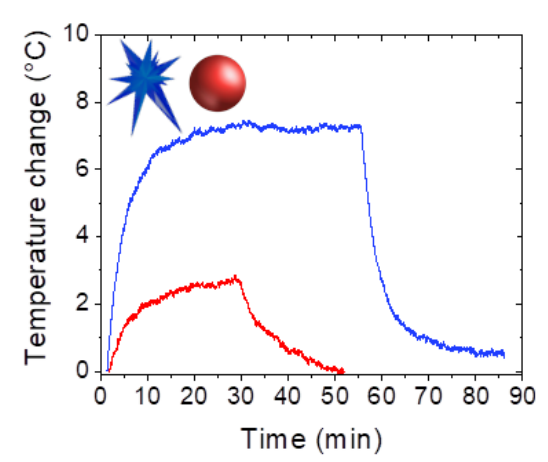

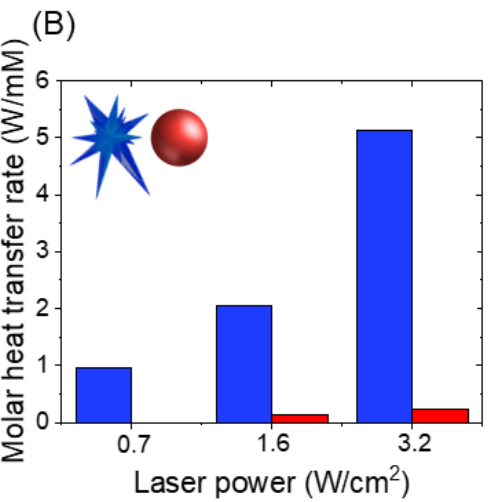

(C)

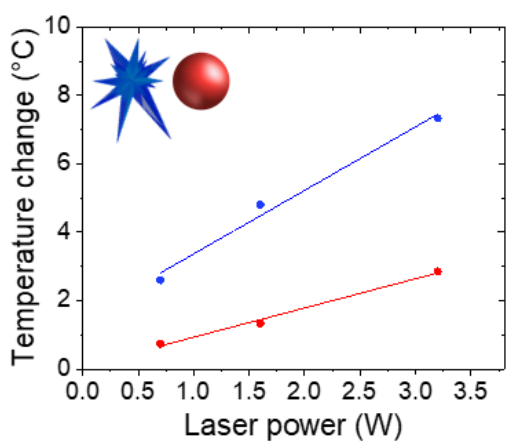

172

173

Figure 3. Plasmonic heating effects of NIR laser $(\lambda=808 \mathrm{~nm})$ on AuNSt@CALB (blue) and AuNSp@CALB (red). (A) Example of heating and cooling curves (laser power $3.2 \mathrm{~W} / \mathrm{cm}^{2}$ ). (B) Molar heat transfer rate vs. laser power. (C) Temperature changes measured in colloidal dispersions of AuNSt@CALB (blue) and AuNSp@CALB (red).

(1)

8

In this context, it is expected that photothermal heating can lower the activation energy of the enzyme, according to the Arrhenius analysis ${ }^{22}$, thereby leading to higher enzyme activity. This effect was further confirmed by activity assays for both free CALB and adsorbed onto Au NPs, under different temperatures, as shown in Figure 4A. Typically, each class of enzyme exhibits an optimal temperature where the highest activity is observed. ${ }^{39}$ Above this value, the activity gradually decreases due to protein denaturation. Free CALB showed an optimal temperature of 55 ${ }^{\circ} \mathrm{C}$ (Figure 4A), in agreement with previously reported data. ${ }^{40}$ At temperatures above $55{ }^{\circ} \mathrm{C}$, free CALB undergoes thermal deactivation and its activity decreases considerably. Conversely, the enzymatic activity increased with temperature, even at values above $55^{\circ} \mathrm{C}$, for AuNSt@CALB and AuNSp@CALB. The preservation of enzyme activity at high temperatures indicates that the adsorption of CALB on Au NPs enhances the enzyme thermal stability. Circular dichroism (CD) spectroscopy studies (Figure 4B and Figure S5) demonstrate the higher conformational stability of enzymes adsorbed on both AuNSt and AuNSp, which explains their higher enzyme activities at temperatures above $55^{\circ} \mathrm{C}$. The observed decrease in mean residue ellipticity (MRE) at $222 \mathrm{~nm}$ 
194 temperatures, as no significant ellipticity changes were observed up to $48{ }^{\circ} \mathrm{C}$. However, the

(A)

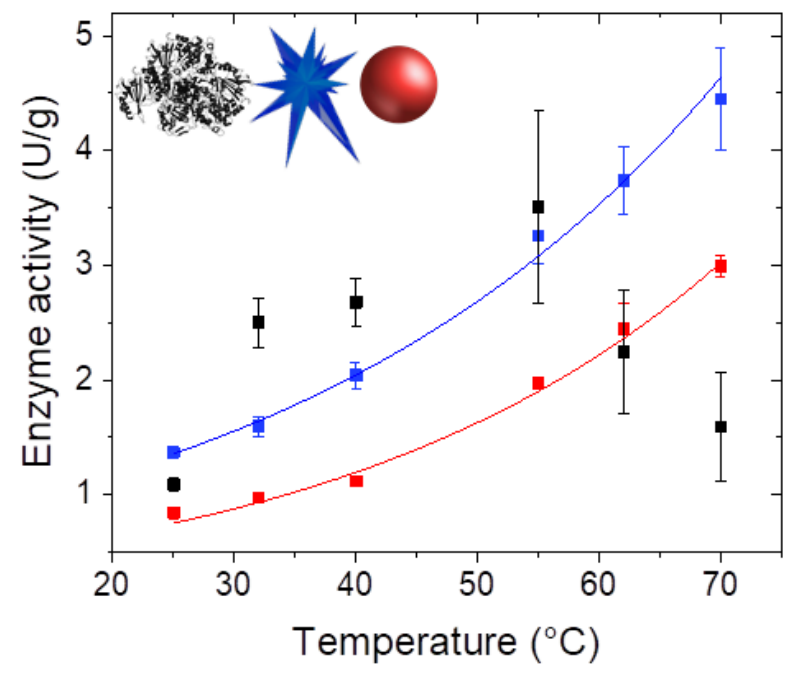

(B)

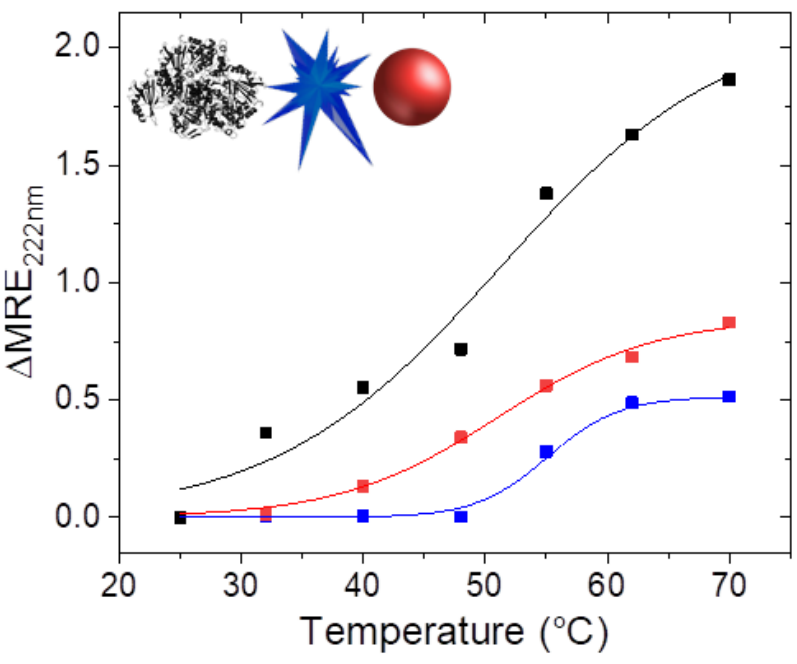

Figure 4. Temperature effects at dark conditions on enzymatic activity (A) and enzyme secondary structure (B), for AuNSt@CALB (blue), AuNSp@CALB (red), and free CALB (black). (A) Enzymatic activity as a function of temperature, fitted to the Arrhenius model. (B) Thermal denaturation of the enzyme conformation monitored by the variation of $\mathrm{MRE}\left(\triangle \mathrm{MRE}=\mathrm{MRE}_{25^{\circ} \mathrm{C}}\right.$ - $\mathrm{MRE}_{\mathrm{T}}$ ) at $222 \mathrm{~nm}$, measured by CD spectroscopy. CD data were obtained by an average of 10 accumulation spectra for each sample. curve for the indirect evaluation of local gradients occurring under irradiation conditions (Figure 2B). This strategy has been previously used to determine the local heating of magnetic iron oxide nanoparticles under alternating magnetic fields. ${ }^{41}$ As presented in Figure 2B, the enzymatic 201 activity of AuNSt@CALB under $3.2 \mathrm{~W} / \mathrm{cm}^{2}$ laser irradiation was 58\% higher than that under non202 irradiated conditions at the same bulk temperature (room temperature). We know that the particles 
203 under $3.2 \mathrm{~W} / \mathrm{cm}^{2}$ laser irradiation are only capable to heat the bulk up to $32{ }^{\circ} \mathrm{C}$ (Figure $3 \mathrm{C}$ ).

204 However, a proportional activity enhancement of $58 \%$ would correspond to a bulk temperature of

205 roughly $42{ }^{\circ} \mathrm{C}$ (see Figure $4 \mathrm{~A}$ ). The differences between the expected activity according to the bulk

206 temperature correlation (Figure 4A) and the measured activity under laser irradiation (Figure 2B)

207 suggest the existence of a local temperature gradient between the AuNSt surface and the bulk. In

208 this way, according to the obtained enzyme activity values, we can estimate a $10^{\circ} \mathrm{C}$ gradient

209 difference between the enzyme environment $\left(42^{\circ} \mathrm{C}\right)$ and the bulk $\left(32^{\circ} \mathrm{C}\right)$. This observation may

210 also be related to previous studies describing the inactivation of enzymes immobilized on Au NPs

211 under laser irradiation, likely due to an excess of local heating. ${ }^{10,11}$

Previous studies have described similar observations on the thermal effects promoted by

213 light absorption. ${ }^{10-15}$ Recently, the effects of photothermal heating and LSPR excited charge

214 carriers were investigated in plasmonic catalysis, ${ }^{4,8,9}$ but a clear distinction of their contributions

215 remains challenging. This is because photothermal heating is largely unavoidable following LSPR

216 excitation. $^{42}$ In the present case, AuNSt seem to release more photothermal heating to the

217 surrounding media as a result of a more efficient LSPR excitation ${ }^{8,43}$ leading to a larger

218 enhancement in the enzyme activity. Moreover, it is plausible that water pocket interfaces present

219 in the enzyme structure can result in higher yields of energy distribution throughout the enzyme

220 structure. $^{44}$ Lastly, we argue that discussing the activity enhancement mechanism through a

221 mechanism based on LSPR-excited charge carriers would be too speculative, as the hydrolysis

222 mechanism does not involve electron transfer and CALB lacks any metallic center that may

223 facilitate electron shuttle. Although both mechanisms might occur simultaneously, electronic

224 effects can be hardly assessed for this system using state-of-the-art methodologies, whereas

225 photothermal effects are more accessible as we showed. 
227 excitation on the activity enhancement observed for AuNSt@CALB through determination of the 228 steady-state kinetic parameters under irradiation conditions. First of all, we investigated how the 229 maximum reaction rate and $\mathrm{k}_{\mathrm{cat}}$ were affected by light irradiation when CALB was adsorbed on 230 Au NPs (Table 1 and Figure S3). The value of the apparent $\mathrm{k}_{\mathrm{cat}}$ is 60\% higher for AuNSt@CALB 231 under irradiation than under non-irradiation conditions. This effect was less noticeable for 232 AuNSp@CALB, and even less for free CALB. Interestingly, $\mathrm{K}_{\mathrm{M}}$ values increased under laser 233 irradiation only when CALB was adsorbed on Au NPs, with no apparent changes in free CALB, 234 suggesting that laser irradiation influences the enzymatic activity when CALB is at the Au NPs 235 surface. The $\mathrm{k}_{\mathrm{cat}} / \mathrm{K}_{\mathrm{M}}$ showed similar values for all samples, regardless of light irradiation, because 236 the effect of light on the catalytic constant is compensated by the effect on the binding constant. 237 The higher $\mathrm{k}_{\mathrm{cat}}$ values under irradiation conditions are probably due to the higher local temperature 238 at the surface of AuNSt, which is also supported by the analysis based on Arrhenius plots (Figure 239 S6). The activation energy barrier for the enzyme activity on AuNSt@CALB decreased from 32 240 to $21 \mathrm{~kJ} \mathrm{~mol}^{-1}$ when the laser was turned on. In contrast, light was unable to alter the activation 241 energy of the free enzyme, supporting that the interface between the enzyme and AuNSt played a 242 key role to enhance the enzymatic activity through plasmonic effects. 244 fundamental mechanistic information for the performance of CALB adsorbed on Au NPs, under 245 light irradiation conditions (Figure 5). According to the general enzymatic mechanism (see Figure 246 6), the reaction kinetics are driven by an initial fast equilibrium binding step followed by an 247 irreversible chemical step. Assuming that the second step of the lipase reaction is the rate-limiting 248 one, the activity assay we used does not account for the product release of the acid, ${ }^{28,45,46}$ since 
249 the colorimetric method only detects the product $p$ NP. The reaction time-courses in Figure 5 were

250 fitted to the initial-burst kinetic model as described by Equation 2, to better estimate the second

251 step of the lipase mechanism. ${ }^{47}$

$$
[P]=v t+\left[E_{0}\right] *\left(1-e^{-k_{o b s} t}\right)
$$

253 Here, the concentration of the formed product $(\mathrm{P})$ is related to the initial velocity $(v)$, the initial 254 concentration of the enzyme $\left(\mathrm{E}_{0}\right)$, and the rate constant $\left(\mathrm{k}_{\mathrm{obs}}\right)$, as a function of time $(\mathrm{t})$ (see Figure 255 S7). In this kinetic model, if $\mathrm{k}_{\mathrm{obs}}>>v, v$ accounts for the rate-limiting step in the enzymatic 256 mechanism of CALB, which we assign to the steps of hydrolysis of the acyl-enzyme complex and 257 product release. Looking beyond the initial burst in the early stages of product conversion with 258 AuNSt@CALB, Figure 5A illustrates that light irradiation affects more significantly the second 259 phase (after ca. 5 min) of the time-courses. Indeed, AuNSt@CALB exhibited a v value which is $26036 \%$ higher under irradiation than under non-irradiation (dark) conditions. Such a light-driven 261 enhancement was higher than that observed for AuNSp@CALB (12\%). The time-course 262 conversion as a function of time for free CALB did not fit this kinetic model, but the Michaelis263 Menten kinetic parameters clearly demonstrate that the activity of the free enzyme was not affected 264 by light irradiation. Consequently, laser irradiation appears to play a relevant role in the rate265 limiting step of the enzyme reaction mechanism. 
(A)

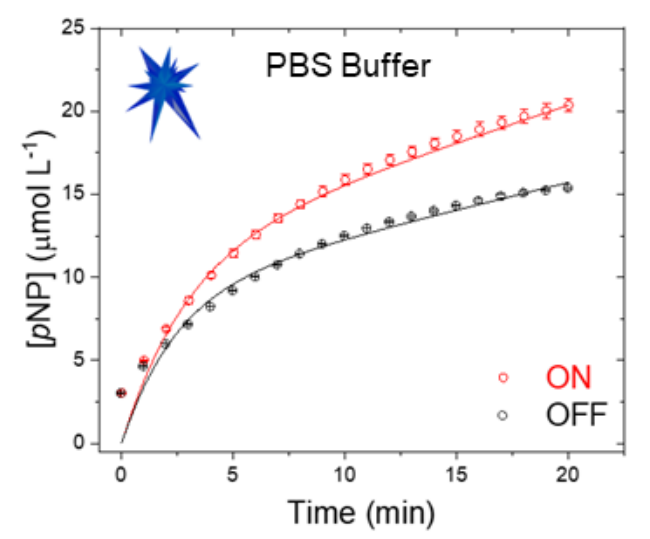

(C)

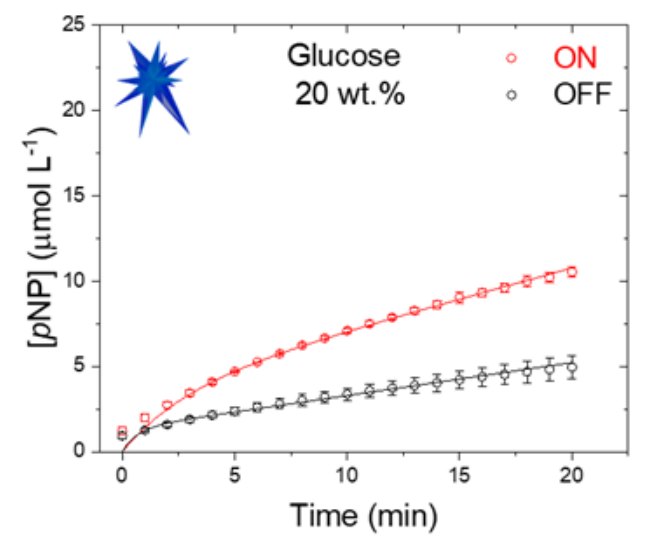

(B)

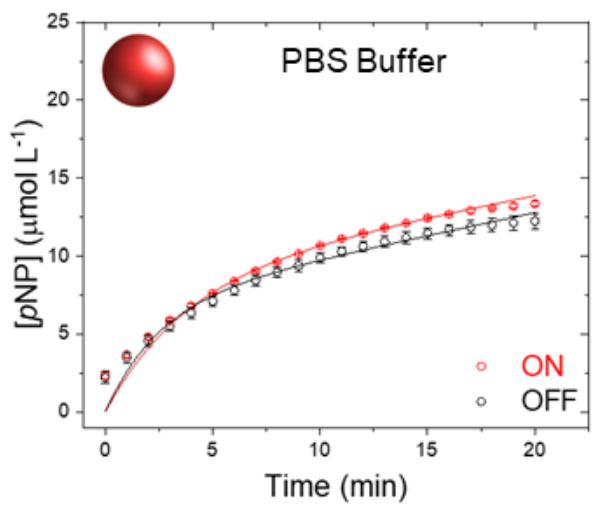

(D)

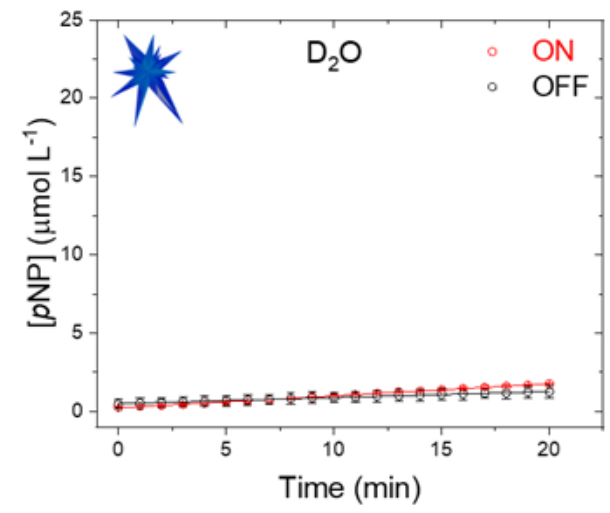

267 Figure 5. Time-courses of pNPP hydrolysis catalyzed in PBS buffer (A and B) by AuNSt@CALB 268 (A, C and D) and AuNSp@CALB (B), under NIR irradiation (3.2 W/ $\left.\mathrm{cm}^{2}\right)$ and non-irradiation 269 (dark) conditions. Viscosity (C) and solvent isotopic (D) effects on the time dependence of the 270 product formation for AuNSt@CALB. Viscosity assays were performed in presence of glucose 20 $271 \mathrm{wt} . \%$ and solvent isotopic assays were performed in the presence of $\mathrm{D}_{2} \mathrm{O}$. All experimental data 272 were fitted to an initial-burst kinetic model (Equation 2) and the respective values are listed in 273 Table S1.

276 inspected the well-known three-step catalytic mechanism of hydrolases (Figure 6). ${ }^{23}, 26,45,48,49$

277 This mechanism is defined by four distinct rate constants $\left(\mathrm{k}_{1}, \mathrm{k}_{-1}, \mathrm{k}_{2}, \mathrm{k}_{3}\right){ }^{26,45,49}$ Therefore, to

278 decipher whether light affects either chemical hydrolysis, product release or both steps, reaction 
280 dark conditions.
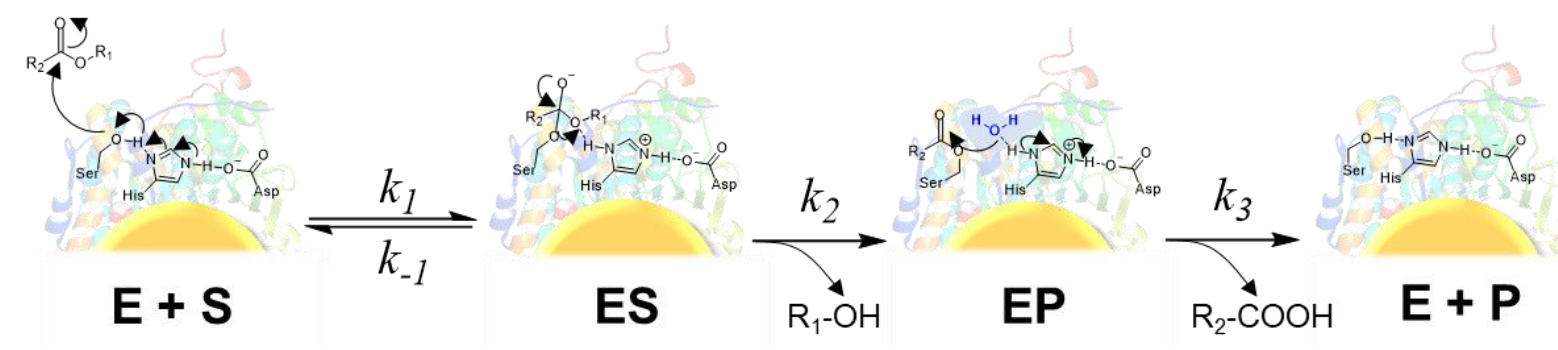

Figure 6. Scheme of the general mechanism for a lipase catalytic reaction. The constants $\mathrm{k}_{1}$ and $\mathrm{k}_{-1}$ are related to reversible binding of the substrate $(\mathrm{S})$ to the enzyme (E) active site, to form the transient intermediate (ES); $\mathrm{k}_{2}$ rules the formation of the acyl-enzyme complex and release of the alcohol product (EP); $\mathrm{k}_{3}$ accounts for the hydrolysis of that complex, releasing the acid product to the bulk $(\mathrm{E}+\mathrm{P})$.

We first monitored reaction time-courses in viscous media (20 wt\% glucose), aiming at hampering the product diffusion out the active center (state $\mathrm{E}+\mathrm{P})$. The interchain hydrogen bonds

290 formed between glucose molecules increase the medium viscosity ${ }^{50}$, like other sugar solutions 291 such as sucrose, ${ }^{22,} 23$ which is known to hamper product release from the enzyme 292 microenvironment. Under these conditions, the product release appears to be the dominant rate293 limiting step, ${ }^{23,48}$ so the effects of laser irradiation on the $v$ parameter of the initial-burst model 294 compared to the corresponding control experiments (no glucose) can be quantified. Under both on 295 and off conditions, more viscous reaction media slowed down product formation along time 296 (Figure 5C and Table S1). Remarkably, light irradiation significantly accelerated (by 40\%) the 297 slowest phase of the time-courses using AuNSt@CALB under viscous conditions. Conversely, the 298 influence of light on the performance of both AuNSp@CALB and free CALB was negligible 299 (Figure S8). To confirm the results extracted from the burst-kinetic model, and considering the 
deacylation of the enzyme as the slower step (i.e., $\mathrm{k}_{3}<<\mathrm{k}_{2}$ ), which means that $\mathrm{k}_{3}$ is the rate-limiting

301 step (i.e., $\mathrm{k}_{\mathrm{cat}}=\mathrm{k}_{3}$ ) as supported by recent computational studies ${ }^{51}$, we constructed the three-step

302 kinetic model showed in Figure 6 using the software COPASI. ${ }^{52}$ This model allowed us to estimate

303 k3 values from the reaction courses obtained with AuNSt@CALB (see Table S2 and Figure S9).

304 We found that $\mathrm{k}_{3}$ follows the same trend as the $v$ parameter calculated from the initial-burst kinetic

305 model. Light increases by 2-fold the $\mathrm{k}_{3}$ value of AuNSt@CALB, compared to the non-irradiated

306 reaction, and the $\mathrm{k}_{3(\mathrm{ON})} / \mathrm{k}_{3(\mathrm{OFF})}$ ratio is maximized under viscous reaction media (in the presence of

307 glucose) by a factor of 3.5 (Figure S10). These experimental results suggest that light contributes

308 to enhancing the catalytic properties of AuNSt@CALB through easing the product release (state

$309 \mathrm{E}+\mathrm{P})$ from enzyme close to the plasmonic NPs surface. Subsequently, to evaluate whether light

310 can also affect the kinetics of the hydrolytic step (state EP), the kinetic isotopic effect (KIE) ${ }^{53}$ was

311 studied by using heavy water $\left(\mathrm{D}_{2} \mathrm{O}\right)$ under laser on and off conditions, and the results were

312 compared to their corresponding control experiments (in $\mathrm{H}_{2} \mathrm{O}$ ). In this step, water molecules from

313 the medium play a crucial role in the nucleophilic attack for cleaving the carbonyl group bond of

314 the acyl-enzyme complex. ${ }^{26,}{ }^{49}$ When using $\mathrm{D}_{2} \mathrm{O}$ as solvent, the enzyme activity dramatically

315 decreased for both conditions, as expected from the occurrence of an isotopic effect in the

316 hydrolysis $\operatorname{step}^{53}$ (Figure 5D). Hence, we observe a KIE of $v\left(\mathrm{H}_{2} \mathrm{O}\right) / v\left(\mathrm{D}_{2} \mathrm{O}\right)$ of 8.67 , which

317 demonstrates that the hydrolysis of the acyl-enzyme complex dominates the rate-limiting step $\left(\mathrm{k}_{3}\right)$.

318 On the other hand, when the reaction time-courses were recorded in the presence of $\mathrm{D}_{2} \mathrm{O}$ and under

319 irradiation conditions, light had a negligible effect on the enzymatic rates. Hence, when the

320 hydrolysis of the acyl-enzyme complex is extremely slow due to isotopic effects, NIR laser

321 irradiation no longer affects the rate-limiting step of the AuNSt@CALB catalysis mechanism.

322 Interestingly, these results indicate that plasmonic excitation hardly affects the kinetics of water 
323 attack, while it significantly increases the efficiency of the product release step. When compared

324 to the activity of soluble CALB measured in PBS buffer, we found a stronger temperature

325 dependence of the free enzyme activity in viscous media, but a weaker dependence in $\mathrm{D}_{2} \mathrm{O}$. These

326 results further support the enhancement of product relase when the enzyme is locally heated at the

327 interface with the irradiated plasmonic nanoparticle (Figure S11). Previous studies reporting that

328 enzyme immobilization on the surface of nanoparticles leads to more significant changes in the

329 product release step in the enzymatic kinetics ${ }^{22,23}$ also support the assumption that the major

330 contribution of light is related to this step. Therefore, our results demonstrate that LSPR excitation

331 increases the activity of AuNSt@CALB by enhancing the kinetics of product release in the last

332 step of the enzyme mechanism driven by $\mathrm{k}_{3}$, as summarized in Figure 6. This effect has been

333 observed exclusively with AuNSt@CALB, confirming that the LSPR of Au NPs must be in

334 resonance with the incident NIR laser wavelength $(808 \mathrm{~nm})$, to exert the effect on enzyme 335 properties.

\section{CONCLUSION}

We used CALB adsorbed on the surface of Au nanospheres (NSp) and nanostars (NSt) as

338 a model system to unravel the effect of light illumination, and thus LSPR excitation, on the

339 underlying mechanisms behind the plasmonic enhancement of enzyme activity under NIR

340 excitation. It was found that LSPR excitation in the NIR enabled an increase of $58 \%$ in enzyme

341 activity when Au NSt were employed as immobilization carriers. In addition to the enhanced

342 activities, we investigated the effect of plasmonic excitation on the rate-limiting step of the

343 enzymatic reaction. Data from highly viscous conditions and solvent isotopic effects revealed that

344 photothermal heating from LSPR excitation accelerated the latest step of the reaction by favoring

345 product release, rather than improving the hydrolytic step at the interface between the enzyme and 
346 the plasmonic NPs. We envision that some of the mechanistic conclusion reached in this work can

347 be translated to other combinations of enzymes and plasmonic NPs, and may inspire the rational

348 design of plasmonic NPs and enzyme hybrids with target activities and selectivity that can be

349 externally controlled by light excitation.

351 Materials. Lipase from Candida antarctica fraction B (CALB), tetrachloroauric acid $352\left(\mathrm{HAuCl}_{4} 3 \mathrm{H}_{2} \mathrm{O}\right)$, sodium citrate tribasic dihydrate, ascorbic acid, silver nitrate, 4-nitrophenyl 353 palmitate were purchased from Sigma-Aldrich. Phosphate-buffered saline was purchased from

354 Biochrom GmbH, (Berlin, Germany). CALB solutions were prepared in PBS buffer $\mathrm{pH}$ 7.4. The 355 concentration of CALB was determined by the colorimetric kit Bradford assay ${ }^{54}$, purchased from 356 Thermo Scientific. All chemicals were used as received. Purified Milli-Q water (Millipore, 18.2 $357 \mathrm{M} \Omega \mathrm{cm}$ ) was used in the preparation of all solutions.

\section{Gold nanoparticles synthesis and CALB adsorption.}

359 AuNSp@CALB synthesis. AuNSp were obtained by Turkevich method. ${ }^{55}$ In a typical procedure, $360150 \mathrm{~mL}$ of $2.2 \mathrm{mmol} \mathrm{L}^{-1}$ sodium citrate solution under vigorous stirring was heated until boiling. 361 Then, $1 \mathrm{~mL}$ of $25 \mathrm{mmol} \mathrm{L}^{-1} \mathrm{HAuCl}_{4} \cdot 3 \mathrm{H}_{2} \mathrm{O}$ was added. The temperature was decreased to $\sim 90{ }^{\circ} \mathrm{C}$ 362 and the solution color change from soft yellow to red in $\sim 10 \mathrm{~min}$. After the solution reach $90^{\circ} \mathrm{C}$,

$3631 \mathrm{~mL}$ of $60 \mathrm{mmol} \mathrm{L}^{-1}$ sodium citrate solution and $1 \mathrm{~mL}$ of $25 \mathrm{mmol} \mathrm{L}^{-1} \mathrm{HAuCl}_{4} \cdot 3 \mathrm{H}_{2} \mathrm{O}$ were 364 subsequently added, and let it stir during $30 \mathrm{~min}$ at $90{ }^{\circ} \mathrm{C}$. After cooling to room temperature, 365 samples were stored in fridge for further use. The adsorption of CALB onto AuNSp to obtain 366 AuNSp@CALB bioconjugates was adapted from a previously described method. ${ }^{24}$ First, $0.1 \mathrm{mg}$ $367 \mathrm{~mL}^{-1}$ CALB stock solutions were prepared in PBS buffer $\mathrm{pH}$ 7.4. $10 \mathrm{~mL}$ of CALB solution was 
368 added to $10 \mathrm{~mL}$ of previously synthesized AuNSp. The sample was incubated during $2 \mathrm{~h}$ at $32{ }^{\circ} \mathrm{C}$

369 and $300 \mathrm{rpm}$ in an Eppendorf thermomixer. After, before using, the colloidal dispersion was

370 washed by centrifugation at $13000 \mathrm{rpm}$ during $20 \mathrm{~min}$ to remove the excess of CALB and possible

371 non-reactants from AuNSp synthesis. The precipitate was washed and re-dispersed in PBS buffer.

372 Samples were previous analyzed by UV-Vis spectroscopy (Agilent 8453) to monitor the LSPR

373 signal and to determine the molar gold concentration in the samples at $\lambda=400 \mathrm{~nm}^{38}$.

374 AuNSp@CALB final concentration of Au was $0.84 \mu \mathrm{mol} \mathrm{L}^{-1}$ and of CALB was $1.06 \mu \mathrm{mol} \mathrm{L}^{-1}$.

375 AuNSt@CALB synthesis. AuNSt@CALB synthesis were adapted from a previously described

376 method. ${ }^{33,34}$ AuNSt were obtained by seed-mediated growth. Firstly, seed solution was prepared

377 by adding $5 \mathrm{~mL}$ of $34 \mathrm{mmol} \mathrm{L}^{-1}$ sodium citrate into $95 \mathrm{~mL}$ of $0.5 \mathrm{mmol} \mathrm{L}^{-1} \mathrm{HAuCl}_{4} .3 \mathrm{H}_{2} \mathrm{O}$ under

378 boiling and vigorous stirring, and it was let stirring during $15 \mathrm{~min}$ at the same temperature and

379 stirring. After cooling to room temperature, the colloidal dispersion was stored in fridge for further

380 use. For AuNSt synthesis, $100 \mu \mathrm{L}$ of $25 \mathrm{mmol} \mathrm{L}^{-1} \mathrm{HAuCl}_{4} \cdot 3 \mathrm{H}_{2} \mathrm{O}$ was added into $10 \mathrm{~mL}$ of $\mathrm{H}_{2} \mathrm{O}$

381 containing $10 \mu \mathrm{L}$ of $1 \mathrm{mmol} \mathrm{L}^{-1} \mathrm{HCl}$ under vigorous stirring at room temperature. Then, $100 \mu \mathrm{L}$

382 of seed solution, $100 \mu \mathrm{L}$ of $3 \mathrm{mmol} \mathrm{L}^{-1}$ silver nitrate solution and $50 \mu \mathrm{L}$ of $100 \mathrm{mmol} \mathrm{L}^{-1}$ ascorbic

383 acid solution were quickly subsequent added. After 3-5 min of stirring, $10 \mathrm{~mL}$ of $0.1 \mathrm{mg} \mathrm{mL}^{-1}$

384 CALB solution was added and let it stirring for $5 \mathrm{~min}$. Then, the sample was stored in fridge for

385 further use. Samples were washed just before the use by centrifugation at $7000 \mathrm{rpm}$ during $15 \mathrm{~min}$

386 to remove the excess of CALB and possible non-reactants from AuNSt synthesis. The precipitate

387 was washed and re-dispersed in PBS buffer. Samples were previous analyzed by UV-Vis

388 spectroscopy (Agilent 8453) to monitor the LSPR signal and to determine the molar gold

389 concentration in the samples at $\lambda=400 \mathrm{~nm}^{38}$. AuNSt@CALB final concentration of Au was 0.54

$390 \mu \mathrm{mol} \mathrm{L}^{-1}$ and of CALB was $1.14 \mu \mathrm{mol} \mathrm{L}^{-1}$. 


\section{$391 \quad$ Enzymatic activity assays.}

392 pNPP hydrolysis time dependence of the product formation. Enzymatic activity of free CALB and

393 CALB-AuNSp bioconjugates were determined by measuring the release of 4-nitrophenolate $(p N P)$

394 from the hydrolysis of 4-nitrophenyl palmitate ( $p$ NPP) (Scheme 1), monitored by UV-Vis 395 spectroscopy at $\lambda=405 \mathrm{~nm}$, as previously described elsewhere. ${ }^{24,26}$ In a quartz cuvette containing $3961000 \mu \mathrm{L}$ of PBS buffer $\mathrm{pH} 7.4,36 \mu \mathrm{L}$ of $0.5 \mathrm{mmol} \mathrm{L}^{-1} p \mathrm{NPP}$ solution previously prepared in 397 isopropanol was added. All $p$ NPP solutions were prepared in the same day before use. 398 Subsequently, $36 \mu \mathrm{L}$ of the sample was added and homogenized. The solution change slowly from 399 transparent to light yellow upon $p$ NP release, according to the amount of CALB in the sample. $400 p$ NP concentration was determined from Lambert-Beer's law using molar extinction coefficient of $401 \varepsilon=12800 \mathrm{~mol} \mathrm{~L}^{-1} \mathrm{~cm}^{-1} \cdot{ }^{26}$ The enzymatic activity was determined from the initial velocity obtained 402 from the linear slope of $p \mathrm{NP}$ concentration versus time plot. The unit $\mathrm{U} / \mathrm{g}$ corresponds to $1 \mu \mathrm{mol}$ 403 of the product $p$ NP formed per 1 min of reaction related to the amount of protein. For the assays 404 upon laser illumination, the cuvette was illuminated vertically (see illustrative scheme in Figure $4052 \mathrm{~A})$ and measures of the absorbance of $p \mathrm{NP}$ formation were recorded at each 1 min during 406 approximately $20 \mathrm{~min}$. 
<smiles>CCCCCCCCCCCCCCCCCCCC(=O)Oc1ccc([O-])cc1</smiles>

4-nitrophenyl palmitate $(p \mathrm{NPP})$

4-nitrophenolate $(p \mathrm{NP})$

408 Scheme 1. Hydrolysis of pNPP biocatalyzed by CALB. The reaction rate can be monitored from 409 pNP formation, by monitoring absorbance at $\lambda=405 \mathrm{~nm}$.

411 Michaelis-Menten. Enzymatic kinetics of free CALB and CALB-AuNPs bioconjugates were 412 determined by the typical procedure of Michaelis-Menten model ${ }^{28,56}$. First, $p$ NPP solutions at 413 initial concentrations of $0.5,0.4,0.3,0.2,0.1,0.05$, and $0.01 \mathrm{mmol} \mathrm{L}^{-1}$ were prepared in 414 isopropanol. All $p$ NPP solutions were prepared at the same day before use. The same procedure 415 described in the previous topic for $p$ NPP hydrolysis time dependence of the product formation to 416 determine the enzyme activity was performed. The values of the parameters maximum velocity $417\left(\mathrm{~V}_{\max }\right)$ and Michaelis-Menten constant $\left(\mathrm{K}_{\mathrm{M}}\right)$, related to the initial velocity $\left(\mathrm{V}_{\mathrm{o}}\right)$ and substrate 418 concentration ([S]), were obtained from the typical relation:

$$
V_{o}=\frac{V_{\max } *[S]}{K_{M}+[S]}
$$

420 Arrhenius. Arrhenius analysis was performed to determine the enzyme activation energy of free

421 CALB and CALB-AuNPs bioconjugates as described previously elsewhere ${ }^{22}$. The enzyme activity 422 was determined by the same procedure described in the former topic for $p$ NPP hydrolysis, varying 423 the temperature from 25 to $80{ }^{\circ} \mathrm{C}$. The values of activation energy $\left(\mathrm{E}_{\mathrm{a}}\right)$ were obtained from the 424 linear fitting from the relation described as 
426 where, $\mathrm{k}$ is the rate constant, $\mathrm{A}$ is the pre-exponential factor, $\mathrm{R}$ is the universal gas constant at the 427 absolute temperature (T).

428 Viscosity and solvent isotope dependence. Viscosity and solvent isotope dependence kinetics were 429 performed as previous described elsewhere. ${ }^{23,48}$ For the viscosity assays, kinetics was performed 430 in presence of $20 \mathrm{wt} \%$ glucose prepared in PBS buffer. For the solvent isotope assays, kinetics was 431 performed in presence of $\mathrm{D}_{2} \mathrm{O}$ and all samples were previously washed and re-suspended in $\mathrm{D}_{2} \mathrm{O}$ 432 to avoid any water molecules at the kinetics. Enzyme activity was determined by the same 433 procedure described in the previous topic for $p$ NPP hydrolysis time dependence of the product 434 formation. Data were analyzed and fitted by an initial-burst of product kinetics model. ${ }^{47}$

435 Heating experiments. $1 \mathrm{~mL}$ of sample in a quartz cuvette was illuminated by a near-infrared laser 436 at $\lambda=808 \mathrm{~nm}$ (fiber-coupled laser diode, Lumics LU0808T040) laterally, passing through two 437 lenses, one to collimate and other to expand the laser beam in order to illuminate a spot of $1 \mathrm{~cm}^{2}$ 438 onto the sample. The laser was illuminated upon different powers $\left(0.7,1.6\right.$, and $\left.3.2 \mathrm{~W} / \mathrm{cm}^{2}\right)$ and 439 monitored by using a thermal camera (FLIR A35) above the cuvette. The heating and cooling 440 curves were obtained from the thermal camera data by using the ResearchIR software. PBS buffer 441 and water were measured as blank curves to eliminate any contribution from the medium. The 442 molar heat rate transfer was calculated by the relation ${ }^{37}$ :

$$
\frac{\Delta Q}{c_{A u}}=\frac{Q_{\text {sample }}-Q_{\text {medium }}}{\varepsilon_{400} / 2.4 \text { mmolL }^{-1}}
$$


444 where, the generated heat output $(\Delta \mathrm{Q})$, obtained from the difference of heat from the sample

$445\left(\mathrm{Q}_{\text {sample }}\right)$ and from the medium $\left(\mathrm{Q}_{\text {medium }}\right)$, is related in terms of the estimate gold concentration $\left(\mathrm{c}_{\mathrm{Au}}\right.$ $\left.446=\varepsilon_{400} / 2.4 \mathrm{mmol} \mathrm{L}^{-1}\right)^{38}$ in the sample.

\section{Characterization techniques.}

448 Transmission electron microscopy (TEM). TEM images were obtained by using a JEOL 449 microscope at an acceleration voltage of $200 \mathrm{kV}$. Approximately $3 \mu \mathrm{L}$ of sample was dropped on 450 a lacey carbon-coated grid and left to dry. The size distribution of nanoparticles obtained were 451 analyzed by using ImageJ software.

452 Circular dichroism (CD) spectroscopy. CD measurements were obtained in a Jasco J-815 CD 453 spectrometer. CD spectra were recorded in the range $200-260 \mathrm{~nm}$, using a quartz cuvette of $5 \mathrm{~mm}$, 454 bandwidth of $5 \mathrm{~nm}$, data pitch of $1 \mathrm{~nm}$, scanning speed at $50 \mathrm{~nm} / \mathrm{min}$. The spectra were obtained 455 by an average of 10 accumulations and corrected by the PBS buffer spectrum. The measurements 456 were showed in molar residue ellipticity (MRE) by using the relation:

$$
M R E=\frac{M R W \times \theta}{10 d C}
$$

458 where, the measured ellipticity $(\theta)$ in degrees is related to the cuvette path length $(d)$ in centimeters 459 and the protein concentration (C) in $\mathrm{g} \mathrm{mL}^{-1}$. MRW corresponds to the mean residue weight defined 460 by $\mathrm{MRW}=\mathrm{M} /(\mathrm{N}-1)$, where $\mathrm{M}$ is the molecular mass in Daltons and $\mathrm{N}$ is the number of amino 461 acids in the protein structure. For CALB, $\mathrm{M}=33000 \mathrm{~g} \mathrm{~mol}^{-1}$ and $\mathrm{N}=317 .^{40}$ 


\section{Supporting Information.}

465 The following files are available free of charge.

466 Additional information of LSPR characterization before and after NIR laser irradiation; Michaelis-

467 Menten plots; NIR laser power effect on free CALB; CD spectra as function of temperature;

468 Arrhenius plots, example of initial-burst of product kinetics; viscosity on time dependence of the

469 product formation; fitting data carried out with COPASI and the values obtained; Table containing

470 parameters obtained from kinetics fitted data and from COPASI software (PDF)

\section{AUTHOR INFORMATION}

\section{Corresponding Author}

473 Heloise R. Barros - Department of Fundamental Chemistry, Institute of Chemistry, University of 474 São Paulo, Av. Prof. Lineu Prestes, 748, Vila Universitária, 05508-000 São Paulo, SP, Brazil; CIC 475 biomaGUNE, Basque Research and Technology Alliance (BRTA), Paseo de Miramón 182, 20014

476 Donostia - San Sebastián, Spain. Email: barroshr@usp.br

477 Fernando López-Gallego - CIC biomaGUNE. Basque Research and Technology Alliance (BRTA), 478 Paseo de Miramón 182, 20014 Donostia - San Sebastián, Spain; Ikerbasque, Basque Foundation 479 for Science, 48013 Bilbao, Spain. Email: flopez@cicbiomagune.es

\section{Author Contributions}

481 The manuscript was written through contributions of all authors. All authors have given approval 482 to the final version of the manuscript.

\section{$483 \quad$ Notes}


484 The authors declare no competing financial interest.

485

486

487

488

489

490

491

492

493

494

\section{ACKNOWLEDGMENT}

Authors thank Brazilian agencies CNPq and São Paulo Research Foundation FAPESP (2015/26308-7, 2018/13492-2) for financial support. HRB also thanks FAPESP for the fellowships granted (2019/09668-0, 2017/20892-4). PHCC thanks FAPESP, the University of Helsinki, and the Jane and Aatos Erkko Foundation for support. CK thanks funding from the European Union's Horizon 2020 research and innovation program under the Marie Skłodowska-Curie grant agreement No. 799393 (NANOBIOME). LML-M and IG acknowledges funding from the Spanish State Research Agency (Grant MAT2017-86659-R). Funding from IKERBASQUE to LML-M and FLG is also acknowledged. This work was performed under the Maria de Maeztu Units of Excellence Program from the Spanish State Research Agency - Grant No. MDM-2017-0720.

\section{ABBREVIATIONS}

Au NPs, gold nanoparticles; AuNSp, gold nanospheres; AuNSt, gold nanostars; CALB, Candida antarctica fraction $\mathrm{B} ; \mathrm{CD}$, circular dichroism; $\mathrm{k}$, rate constant; $\mathrm{k}_{\mathrm{cat}}$, apparent catalytic rate; $\mathrm{k}_{\mathrm{cat}} / \mathrm{K}_{\mathrm{M}}$, catalytic efficiency; KIE, kinetic isotopic effect; $\mathrm{K}_{\mathrm{M}}$, Michaelis constant; LSPR, localized surface plasmon resonance; MRE, mean residue ellipticity; NIR, near-infrared; $p$ NP, 4-nitrophenolate; pNPP, 4-nitrophenyl palmitate; TEM, transmission electron microscopy.

\section{REFERENCES}

1. Mosquera, J.; Zhao, Y.; Jang, H. J.; Xie, N. L.; Xu, C. L.; Kotov, N. A.; Liz-Marzán, L. M., Plasmonic Nanoparticles with Supramolecular Recognition. Adv. Funct. Mater. 2020, 30, 117.

2. Litti, L.; Reguera, J.; de Abajo, F. J. G.; Meneghetti, M.; Liz-Marzán, L. M., Manipulating Chemistry Through Nanoparticle Morphology. Nanoscale Horiz. 2020, 5, 102-108. 
3. Bodelón, G.; Costas, C.; Pérez-Juste, J.; Pastoriza-Santos, I.; Liz-Marzán, L. M., Gold Nanoparticles for Regulation of Cell Function and Behavior. Nano Today 2017, 13, 40-60.

510 4. Liz-Marzán, L. M.; Murphy, C. J.; Wang, J. F., Nanoplasmonics. Chem. Soc. Rev. 2014, $511 \quad 43,3820-3822$.

512 5. Araujo, T. P.; Quiroz, J.; Barbosa, E. C. M.; Camargo, P. H. C., Understanding Plasmonic 513 Catalysis with Controlled Nanomaterials Based on Catalytic and Plasmonic Metals Curr. Opin. 514 Colloid Interface Sci. 2019, 39, 110-122.

515 6. Linic, S.; Aslam, U.; Boerigter, C.; Morabito, M., Photochemical Transformations on 516 Plasmonic Metal Nanoparticles. Nat. Mat. 2015, 14, 567-576.

517 7. Wang, H.; Liu, T.; Huang, Y. Z.; Fang, Y. R.; Liu, R. C.; Wang, S. X.; Wen, W. J.; 518 Sun, M. T., Plasmon-Driven Surface Catalysis in Hybridized Plasmonic Gap Modes. Sci. Rep. 519 2014, 4.

$520 \quad 8 . \quad$ Baffou, G.; Quidant, R., Thermo-Plasmonics: Using Metallic Nanostructures as NanoSources of Heat. Laser \& Photonics Rev. 2013, 7, 171-187.

9. Baffou, G.; Quidant, R., Nanoplasmonics for Chemistry. Chem. Soc. Rev. 2014, 43, 38983907.

10. Guo, S. J.; Li, H.; Liu, J.; Yang, Y. M.; Kong, W. Q.; Qiao, S.; Huang, H.; Liu, Y.; Kang, Z. H., Visible-Light-Induced Effects of Au Nanoparticle on Laccase Catalytic Activity. ACS Appl. Mater. Interfaces 2015, 7, 20937-20944.

11. Bretschneider, J. C.; Reismann, M.; von Plessen, G.; Simon, U., Photothermal Control of the Activity of HRP-Functionalized Gold Nanoparticles. Small 2009, 5, 2549-2553.

12. Blankschien, M. D.; Pretzer, L. A.; Huschka, R.; Halas, N. J.; González, R.; Wong, M. S., Light-Triggered Biocatalysis Using Thermophilic Enzyme-Gold Nanoparticle Complexes. ACS Nano 2013, 7, 654-663.

13. Li, W.; Liu, D. N.; Geng, X.; Li, Z. Q.; Gao, R. J., Real-Time Regulation of Catalysis by Remote-Controlled Enzyme-Conjugated Gold Nanorod Composites for Aldol Reaction-Based Applications. Catal. Sci. Technol. 2019, 9, 2221-2230.

14. Tadepalli, S.; Yim, J.; Madireddi, K.; Luang, J. Y.; Naik, R. R.; Singamaneni, S., Gold Nanorod-Mediated Photothermal Enhancement of the Biocatalytic Activity of a PolymerEncapsulated Enzyme. Chem. Mater. 2017, 29, 6308-6314.

15. Tadepalli, S.; Yim, J.; Cao, S. S.; Wang, Z. Y.; Naik, R. R.; Singamaneni, S., MetalOrganic Framework Encapsulation for the Preservation and Photothermal Enhancement of Enzyme Activity. Small 2018, 14.

16. Yang, S. Y.; Yao, D. F.; Wang, Y. S.; Yang, W. T.; Zhang, B. B.; Wang, D. B., EnzymeTriggered Self-Assembly of Gold Nanoparticles for Enhanced Retention Effects and Photothermal Therapy of Prostate Cancer. Chem. Commun. 2018, 54, 9841-9844.

17. Khiavi, M. A.; Safary, A.; Aghanejad, A.; Barar, J.; Rasta, S. H.; Golchin, A.; Omidi, Y.; Somi, M. H., Enzyme-Conjugated Gold Nanoparticles for Combined Enzyme and Photothermal Therapy of Colon Cancer Cells. Colloids Surf. A 2019, 572, 333-344.

18. Yang, K. K.; Liu, Y. J.; Wang, Y.; Ren, Q. L.; Guo, H. Y.; Matson, J. B.; Chen, X. Y.; Nie, Z. H., Enzyme-Induced in Vivo Assembly of Gold Nanoparticles for Imaging-Guided Synergistic Chemo-Photothermal Therapy of Tumor. Biomaterials 2019, 223.

19. Barros, H. R.; López-Gallego, F.; Liz-Marzán, L. M., Light-Driven Catalytic Regulation of Enzymes at the Interface with Plasmonic Nanomaterials. Biochemistry 2020, Article ASAP. 
20. Nel, A. E.; Madler, L.; Velegol, D.; Xia, T.; Hoek, E. M. V.; Somasundaran, P.; Klaessig, F.; Castranova, V.; Thompson, M., Understanding Biophysicochemical Interactions at the Nano-Bio Interface. Nat. Mater. 2009, 8, 543-557.

21. Ansari, S. A.; Husain, Q., Potential Applications of Enzymes Immobilized on/in Nano Materials: A Review. Biotechnol. Adv. 2012, 30, 512-523.

22. Breger, J. C.; Ancona, M. G.; Walper, S. A.; Oh, E.; Susumu, K.; Stewart, M. H.; Deschamps, J. R.; Medintz, I. L., Understanding How Nanoparticle Attachment Enhances Phosphotriesterase Kinetic Efficiency. ACS Nano 2015, 9, 8491-8503.

23. Breger, J. C.; Oh, E.; Susumu, K.; Klein, W. P.; Walper, S. A.; Ancona, M. G.; Medintz,

I. L., Nanoparticle Size Influences Localized Enzymatic Enhancement-A Case Study with

24. de Barros, H. R.; Santos, M. C.; Barbosa, L. R. S.; Piovan, L.; Riegel-Vidotti, I. C., Physicochemical Study of the Interaction between Gold Nanoparticles and Lipase from Candida sp. (CALB): Insights into the Nano-Bio Interface. J. Braz. Chem. Soc. 2019, 30, 2231-2242.

25. Kisukuri, C. M.; Palmeira, D. J.; Rodrigues, T. S.; Camargo, P. H. C.; Andrade, L. H., Bimetallic Nanoshells as Platforms for Metallo- and Biometallo-Catalytic Applications. Chemcatchem 2016, 8, 171-179.

26. Wu, C. S.; Wu, C. T.; Yang, Y. S.; Ko, F. H., An Enzymatic Kinetics Investigation into the Significantly Enhanced Activity of Functionalized Gold Nanoparticles. Chem. Commun. 2008, 5327-5329.

27. Baumann, V.; Muhammed, M. A. H.; Blanch, A. J.; Dey, P.; Rodriguez-Fernandez, J., Biomolecules in Metal and Semiconductor Nanoparticle Growth. Isr. J. Chem. 2016, 56, 195-213. 28. Johnson, B. J.; Algar, W. R.; Malanoski, A. P.; Ancona, M. G.; Medintz, I. L., Understanding Enzymatic Acceleration at Nanoparticle Interfaces: Approaches and Challenges. Nano Today 2014, 9, 102-131.

29. Song, Y. H.; Chen, J. Y.; Liu, H. Y.; Song, Y. G.; Xu, F. G.; Tan, H. L.; Wang, L., Conformation, Bioactivity and Electrochemical Performance of Glucose Oxidase Immobilized on Surface of Gold Nanoparticles. Electrochim. Acta 2015, 158, 56-63.

30. Lopez-Tobar, E.; Hernandez, B.; Ghomi, M.; Sanchez-Cortes, S., Stability of the Disulfide Bond in Cystine Adsorbed on Silver and Gold Nanoparticles As Evidenced by SERS Data. Journal of Physical Chemistry C 2013, 117 (3), 1531-1537.

31. Hakkinen, H., The Gold-Sulfur Interface at the Nanoscale. Nat. Chem. 2012, 4, 443-455.

32. Irani, M.; Tornvall, U.; Genheden, S.; Larsen, M. W.; Hatti-Kaul, R.; Ryde, U., Amino Acid Oxidation of Candida antarctica Lipase B Studied by Molecular Dynamics Simulations and Site-Directed Mutagenesis. Biochemistry 2013, 52, 1280-1289.

33. de Aberasturi, D. J.; Serrano-Montes, A. B.; Langer, J.; Henriksen-Lacey, M.; Parak, W. J.; Liz-Marzán, L. M., Surface Enhanced Raman Scattering Encoded Gold Nanostars for Multiplexed Cell Discrimination. Chem. Mater. 2016, 28, 6779-6790.

34. Yuan, H. K.; Khoury, C. G.; Hwang, H.; Wilson, C. M.; Grant, G. A.; Vo-Dinh, T., Gold Nanostars: Surfactant-Free Synthesis, 3D Modelling, and Two-Photon Photoluminescence Imaging. Nanotechnology 2012, 23.

35. Turkevich, J.; Stevenson, P. C.; Hillier, J., A Study of the Nucleation and Growth Processes in the Synthesis of Colloidal Gold. Discuss. Faraday Soc. 1951, 55-75.

36. Kheirolomoom, A.; Khorasheh, F.; Fazelinia, H., Influence of External Mass Transfer Limitation on Apparent Kinetic Parameters of Penicillin G Acylase Immobilized on Nonporous Ultrafine Silica Particles. J. Biosci. Bioeng. 2002, 93, 125-129. 
37. Kuttner, C.; Holler, R. P. M.; Quintanilla, M.; Schnepf, M. J.; Dulle, M.; Fery, A.; LizMarzan, L. M., SERS and Plasmonic Heating Efficiency from Anisotropic Core/Satellite Superstructures. Nanoscale 2019, 11, 17655-17663.

38. Hendel, T.; Wuithschick, M.; Kettemann, F.; Birnbaum, A.; Rademann, K.; Polte, J., In Situ Determination of Colloidal Gold Concentrations with UV-Vis Spectroscopy: Limitations and Perspectives. Anal. Chem. 2014, 86, 11115-11124.

39. Daniel, R. M.; Danson, M. J., Temperature and the Catalytic Activity of Enzymes: A Fresh Understanding. FEBS Letters 2013, 587, 2738-2743.

40. Rabbani, G.; Ahmad, E.; Khan, M. V.; Ashraf, M. T.; Bhat, R.; Khan, R. H., Impact of Structural Stability of Cold Adapted Candida antarctica Lipase B (CALB): In Relation to pH, Chemical and Thermal Denaturation. RSC Adv. 2015, 5, 20115-20131.

41. Armenia, I.; Bonavia, M. V. G.; De Matteis, L.; Ivanchenko, P.; Martra, G.; Gornati, R.; de la Fuente, J. M.; Bernardini, G., Enzyme Activation by Alternating Magnetic Field: Importance of the Bioconjugation Methodology. J. Colloid Interface Sci. 2019, 537, 615-628.

42. Jain, P. K., Taking the Heat Off of Plasmonic Chemistry. J. Phys. Chem. C 2019, 123, 24347-24351.

43. Chatterjee, H.; Rahman, D. S.; Sengupta, M.; Ghosh, S. K., Gold Nanostars in Plasmonic Photothermal Therapy: The Role of Tip Heads in the Thermoplasmonic Landscape. J. Phys. Chem. C 2018, 122, 13082-13094.

44. Leitner, D. M.; Pandey, H. D.; Reid, K. M., Energy Transport across Interfaces in Biomolecular Systems. J. Phys. Chem. B 2019, 123, 9507-9524.

45. Johnson, K. A., A Century of Enzyme Kinetic Analysis, 1913 to 2013. FEBS Letters 2013, 587, 2753-2766.

46. Powers, K. T.; Washington, M. T., Analyzing the Catalytic Activities and Interactions of Eukaryotic Translesion Synthesis Polymerases. $1^{\text {st }}$ edition; Academic Press 2017, Vol. 592.

47. Hammes, G.; Hammes-Schiffer, S., Physical Chemistry for the Biological Sciences. 2nd edition ed.; John Wiley \& Sons,: New Jersey, 2015; Vol. 55.

48. Gadda, G.; Fitzpatrick, P. F., Solvent Isotope and Viscosity Effects on the Steady-State Kinetics of the Flavoprotein Nitroalkane Oxidase. FEBS Letters 2013, 587, 2785-2789.

49. Jaeger, K. E.; Dijkstra, B. W.; Reetz, M. T., Bacterial Biocatalysts: Molecular Biology, Three-Dimensional Structures, and Biotechnological Applications of Lipases. Annu. Rev. Microbiol. 1999, 53, 315-351.

50. Telis, V. R. N.; Telis-Romero, J.; Mazzotti, H. B.; Gabas, A. L., Viscosity of Aqueous Carbohydrate Solutions at Different Temperatures and Concentrations. Int. J. Food Prop. 2007, 10, 185-195.

51. Galmes, M. A.; Garcia-Junceda, E.; Swiderek, K.; Moliner, V., Exploring the Origin of Amidase Substrate Promiscuity in CALB by a Computational Approach. ACS Catal. 2020, 10, 1938-1946.

52. Hoops, S.; Sahle, S.; Gauges, R.; Lee, C.; Pahle, J.; Simus, N.; Singhal, M.; Xu, L.; Mendes, P.; Kummer, U., COPASI- A COmplex PAthway SImulator. Bioinformatics 2006, 22, 3067-3074.

53. Quinn, D. M., Solvent Isotope Effects for Lipoprotein-Lipase Catalyzed-Hydrolysis of Water-Soluble para-Nitrophenyl Esters. Biochemistry 1985, 24, 3144-3149.

54. Bradford, M. M., Rapid and Sensitive Method for Quantitation of Microgram Quantities of Protein Utilizing Principle of Protein-Dye Binding. Anal. Biochem. 1976, 72, 248-254. 
643 55. Bastus, N. G.; Comenge, J.; Puntes, V., Kinetically Controlled Seeded Growth Synthesis 644 of Citrate-Stabilized Gold Nanoparticles of up to $200 \mathrm{~nm}$ : Size Focusing versus Ostwald Ripening. 645 Langmuir 2011, 27, 11098-11105.

646 56. Johnson, K. A.; Goody, R. S., The Original Michaelis Constant: Translation of the 1913 647 Michaelis-Menten Paper. Biochemistry 2011, 50, 8264-8269.

648

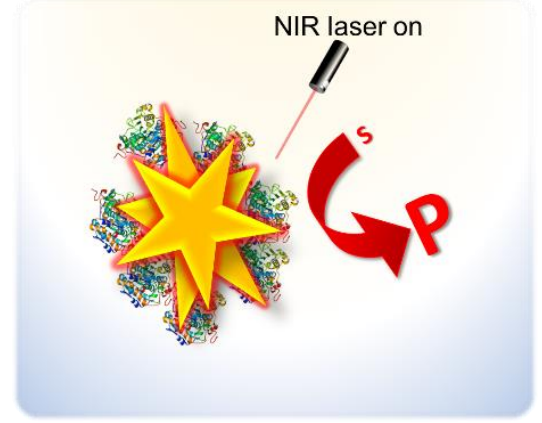

651 Plasmonic biocatalysis: control of enzyme activity by LSPR excitation of plasmonic 652 nanoparticles using external light irradiation. 